\title{
Improving Temporal Resolution in Ambient Noise Monitoring of Seismic Wave Speed
}

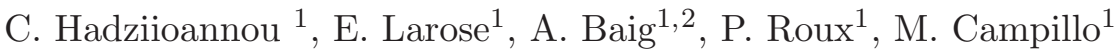

\begin{abstract}
The use of ambient seismic noise has been intensively investigated to perform passive tomography at various scales. Besides passive tomography, passive monitoring is another application of seismic noise correlation as was shown by the recent observation of postseismic velocity changes around the San Andreas Fault in Parkfield, California. One of the drawbacks of using ambient noise correlation for passive monitoring is the need to average the correlations over a long time period in order to obtain a sufficient signal-to-noise ratio (SNR) for the phase fluctuations to be measured accurately. For the application to passive monitoring, one wants the possibility of following short-term velocity variations (one day or less) using noise correlation functions calculated on short time windows. Another difficulty may then appear when the spatial distribution of noise sources also evolves with time. The aim of this paper is to introduce an adaptive filter to the Parkfield dataset in order to improve the SNR output of the ambient noise correlation functions. When applied to passive monitoring, the temporal resolution can be increased from 30 days up to 1 day. With this improved temporal resolution, the velocity drop observed at Parkfield is shown to be cosesimic with the September 24, $2004 M_{w}=6.0$ event. The relationship between the measured velocity fluctuations and the time-evolution of the spatial distribution of the noise wavefield is also investigated. Finally, the error bar in the amplitudes of the velocity variations are compared with a theoretical expectation.
\end{abstract}

\section{Introduction}

It was observed that the two-point cross-correlation between long records of ambient seismic noise yields the Green Function (GF) between the two stations (e.g. Shapiro and Campillo (2004)). This property is expected when noise sources are distributed isotropically around the stations which is

\footnotetext{
${ }^{1}$ Institut des Sciences de la Terre, Université Joseph Fourier and CNRS, BP53, 38041 Grenoble Cedex 9, France

${ }^{2}$ Now at: ESG Solutions, 20 Hyperion Court, Kingston, ON, K7K 7K2, Canada
} 
eventually the case when the ambient noise correlation is integrated over a long time (Weaver and Lobkis (2004); Sanchez-Sesma and Campillo (2006); Wapenaar (2004); Roux et al. (2004)). However, these optimal conditions are almost never fulfilled in seismology as the noise sources (primarily oceanic, Gerstoft and Tanimoto (2007); Landès et al. (2010)) are distributed unevenly. The consequence is that the Green function is only partially reconstructed, and that a certain amount of unpredicted fluctuations remain in the long-time averaged noise correlation. However, using dense network of seismic stations, the large number of correlation pairs provides enough redundancy from the data to counterbalance the relative inaccuracy of traveltime measurements extracted from noise correlation. As a matter of fact, seismic tomography based on noise correlation has been performed successfully at various scales ranging from kilometers to hundred of kilometers (Shapiro et al. (2005)).

Another application of seismic noise correlation is the monitoring of seismic velocity variations. Indeed, an advantage of using noise correlation to retrieve the Green function is that it is a continuous measurement. This allows to track temporal changes from a set of correlation functions calculated on successive time windows of noise recordings. For example, by looking at small phase changes in the signals extracted from noise correlation, we may detect velocity changes occurring in the medium sampled by the seismic waves. In practice, the coda portion of the noise correlation is used for measuring seismic velocity changes. The coda waves have been scattered throughout the medium, and have accumulated the delays due to a velocity change along their trajectories. This makes them more suitable to measuring velocity variations than the direct waves.

What makes this use of noise correlation even more appealing is the work performed by Hadziioannou et al. (2009) through laboratory experiments that showed that passive monitoring was successful without completely reconstructing the GF from cross-correlations. This means that, contrarily to passive imaging, passive monitoring would be viable with a few stations and an uneven distribution of noise sources.

Actually, two ingredients are necessary for passive monitoring. First, measuring accurate phase variations between similar signals requires a good signal-to-noise (SNR) ratio. In particular, the velocity variations associated with earthquakes are very subtle - of the order of $0.1 \%$ relative change - except for the non-linear response of unconsolidated materials at the surface (Peng and Ben-Zion (2006); Rubinstein et al. (2007); Niu et al. (2003)) or in highly damaged fault zones ( $\mathrm{Li}$ et al. (2007)). Since the coda waves strongly contribute to the accuracy of phase variation measurements, the challenge is therefore to obtain a good SNR on this part of the correlation function that is classically of much lower amplitudes than the direct waves. Second, since the spatial distribution of seismic noise is uneven, the correlation function may evolve with time as the noise sources are modified (Froment et al. 
Table 1: Overview of the different timescales used in this paper.

\begin{tabular}{l|l|l}
\hline designation & typical duration & definition \\
\hline $\mathrm{T}$ & $0.1-1 \mathrm{~s}$ & Dominant period of the correlated signal \\
$\mathrm{t}$ & $10-120 \mathrm{~s}$ & Lapse time of the correlated signal \\
$T_{\mathrm{SNR}}$ & $1-30$ days & Averaging time of cross-correlation; stack length. \\
$T_{\text {noise }}$ & days - months & Evolution timescale of the noise sources \\
$T_{\text {medium }}$ & months - years & Evolution timescale of crustal properties \\
\hline
\end{tabular}

(2010)). For example, seasonal changes of ocean noise directivity have been observed, whose effect on the direct arrivals of the noise correlation function is comparable or of greater amplitude than an actual change of the medium velocity.

Going back to the SNR issue, improving SNR in the seismic noise correlation function means averaging over longer noise time series of typically weeks or months (Larose et al. (2008); Sabra et al. (2005)). This need for long averages might be problematic though, since we would like to follow short term variations in the medium as well. In particular, Brenguier et al. (2008a) have measured the velocity changes occurring around an array near Parkfield, California, in the period spanning from 2003-2007. They observed drops in seismic velocity around the array which seem to coincide with the $2004 M_{w}=6.0$ Parkfield earthquake, as well as with the $2003 M_{w}=6.5 \mathrm{San}$ Simeon earthquake. However, since the noise was averaged over 30 days to retrieve the Green function, it is very difficult to say if the observed changes are coseismic or slightly delayed or advanced.

As for the time evolution of the noise source distribution, one may claim that the coda part of the noise correlation function will be less sensitive to the spatial distribution of the noise sources than the direct waves, as was experimentally shown at the laboratory scale (Hadziioannou et al. (2009)). In conclusion, the key issue for passive monitoring is the combination of a strong SNR on strongly-scattered waves extracted from noise correlation. To reach such condition, different timescales play a role in the success of passive monitoring which will be discussed throughout the paper (see table $1): T_{\mathrm{SNR}}$ is the averaging time of the noise correlation function, $T_{\text {medium }}$ is the time evolution of the medium properties (the local velocity change after an earthquake, for example) and $T_{\text {noise }}$ is the time evolution of the spatial distribution of the noise sources.

In this paper, we revisit the 2003-2007 Parkfield data introducing a new processing algorithm with the objective to improve the temporal resolution of the velocity change measurements. The goal of the processing is to improve the SNR of our signals while keeping short temporal averages. The paper is structured as follows: in the next section (section 2), we discuss the data set and the signal processing methods previously applied on the 
noise data. Then, (section 3), we introduce an adaptive filter to improve the SNR of noise-extracted correlation functions, which increases the temporal resolution of velocity variation measurements (sections 4 and 5). We then investigate if the origin of the velocity variations can be attributed to changes in the ambient noise source distribution (section 6). Finally, in section 7 we introduce an expression for estimating the errors made on velocity variation measurements. Conclusion is that the velocity drop observed with waves in the band from 0.1 to $0.9 \mathrm{~Hz}$ at the Parkfield earthquake is coseismic, and that the distribution of the noise source directions has no influence on the velocity variations observed when using the coda of the signal.

\section{Parkfield Data}

The data used in this study is recorded at the Berkeley High Resolution Seismic Network (HRSN ${ }^{3}$ ) near Parkfield, California. This network consists of 13 borehole stations which have been recording continuously since July 2001. The borehole depths range from $60 \mathrm{~m}$ to $300 \mathrm{~m}$, which helps reduce surface effects such as temperature variations and precipitation. The location of the stations and these two earthquakes is shown in Figure 1. For each pair of stations, we consider the vertical component of the continuous record between the 1st of August 2003 and December 31st, 2006. In this period, the $M_{W}=6.5$ San Simeon earthquake occurred on 22 December 2003, as well as the $M_{W}=6.0$ Parkfield earthquake of September 28, 2004. The noise signal recorded at each station is whitened between $\left[\begin{array}{ll}0.1 & 0.9\end{array}\right] \mathrm{Hz}$ using a tapered boxcar window in the frequency domain, and subsequently 1-bit digitized. The daily cross-correlation is then computed for each of the 78 station pairs as well as the 13 autocorrelations, for lapse times between -120 and +120 seconds.

Initially, in an effort to improve the SNR, the daily cross-correlations are stacked with a sliding window of 30 days, which is shifted 1 day at a time. Later on, sliding window stacks of 1 and 5 days are used as well. An example of the signals obtained for a 30 day sliding window for one station pair (JCNB-SMNB) is shown in Figure 2. For each station pair, we define a reference correlation as the average of the correlations for the whole 1248 day measurement period. The temporal evolution of the seismic velocity can be measured by comparing the coda of the reference correlation to that of the shorter stacks. Temporal variations are measured using two different methods, as detailed in section 4 .

Seasonal variations are visible on the ballistic waves (Figure 2, center) of the cross-correlation as an asymmetric change in intensity. This suggests a seasonal change in noise source, as will be investigated in section 6 . Note

\footnotetext{
${ }^{3}$ More information concerning the Parkfield HRSN can be found at http://seismo.berkeley.edu/bdsn/hrsn.overview.html
} 


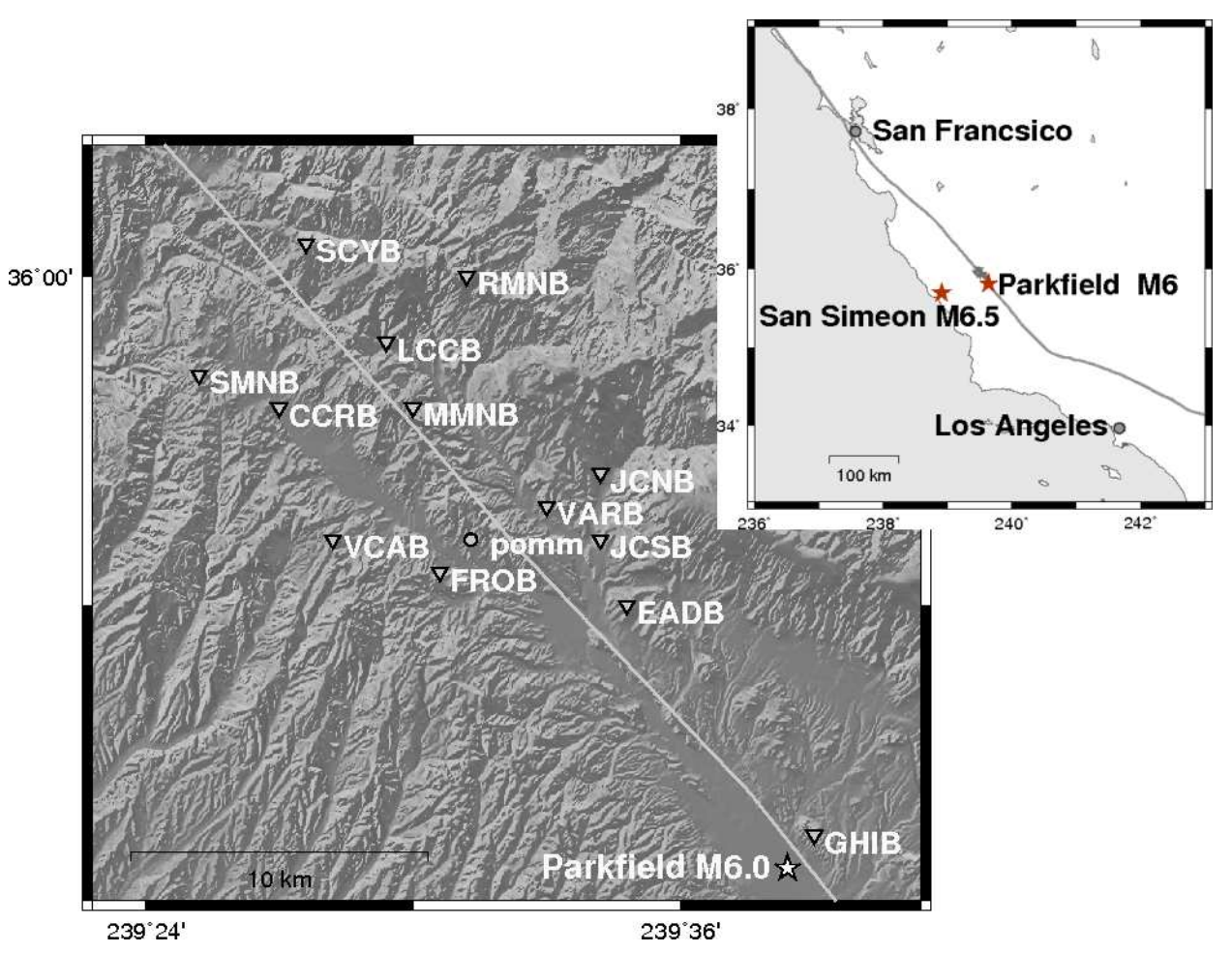

Figure 1: Location of the HRSN stations (white and black triangles) near Parkfield, California. In the inset figure, the locations of the 2003 San Simeon and 2004 Parkfield earthquakes (stars). The white and black circle indicates the location of the GPS station pomm. 


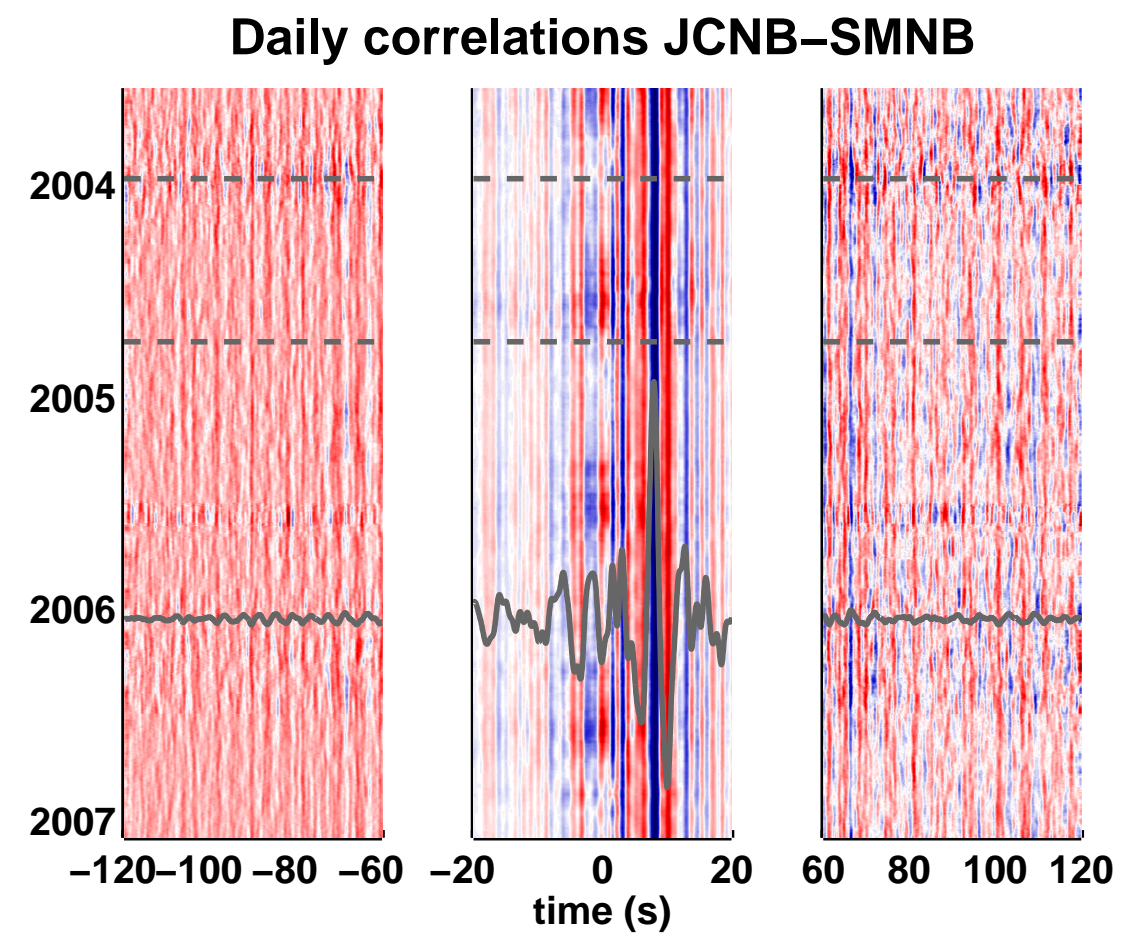

Figure 2: Cross correlation signal for JCNB-SMNB, stacked with a 30-day sliding window. Overlain in gray is the reference trace for this station pair. The gray horizontal dashed lines represent the dates of the San Simeon earthquake (22 December,2003) and the Parkfield earthquake (28 September, 2004). In the center a zoom on the direct waves, on either side on the coda part of the correlations. Note that the seasonal variation visible on the direct waves is no longer present in the coda. 
that this intensity variation is not visible in the coda part of the signal (Figure 2, left and right).

\section{Method: Adaptive Filter}

In an attempt to improve the SNR of the noise correlations, thereby increasing the temporal resolution, an adaptive filter (developed by Baig et al. (2009)) is applied to the correlations before retrieving the velocity variations.

\subsection{The S-transform}

The algorithm used is based on the S-transform, as developed by Stockwell (Stockwell et al. (1996)). The analytical S-transform of a continuous function of time $x(t)$, noted as $S\{x\}(\tau, f)$, is defined as:

$$
S\{x\}(\tau, f)=\frac{1}{\sqrt{2 \pi}} \int_{-\infty}^{\infty} x(t)|f| \exp \left[-\frac{(\tau-t)^{2} f^{2}}{2}-i 2 \pi f t\right] d t,
$$

which transforms the signal $x(t)$ into a time-frequency domain $(\tau, f)$.

The Gaussian envelope function $\left(\exp \left[-(\tau-t)^{2} f^{2} / 2\right]\right)$ localizes in time, and is translated along the signal. In the meantime, the oscillatory exponential kernel $\exp [-i 2 \pi f t]$ fixes the frequency being analyzed, and does not translate. This allows the S-transform to find the amplitude and phase independently. In other words, the S-transform not only estimates the local power spectrum, but also the local phase spectrum.

For a discrete, real signal $x(t)$ with $N$ points, the S-transform will result in $N^{2} / 2$ complex-valued points (the positive frequencies). The S-transforms therefore needs to be evaluated at $N^{2} / 2$ points in time-frequency space. Stockwell (2007) developed the Discrete Orthonormal S-transform (DOST) to reduce the number of evaluations necessary. The $N$-point signal is projected onto a series of $N$ complex basis functions $x_{k}[\tau, f] ; k=1: N$, with each function mapping to a localized patch of time-frequency space described by its frequency, $f$, and a time lag, $\tau$. These basis functions form an orthonormal set. Like its parent S-transform, DOST allows both straightforward manipulation of signals from time to time-frequency while preserving the phase of the signal.

Both the analytical S-transform and the DOST are linear, which is beneficial in application to noise discrimination. Modelling a recorded time series, $x(t)$ as signal, $g(t)$, corrupted with noise, $n(t)$, (ie. $x(t)=g(t)+n(t)$ ), the Stransform of the signal $(S\{x\}(\tau, f))$ becomes:

$$
S\{x\}(\tau, f)=S\{g\}(\tau, f)+S\{n\}(\tau, f)
$$

Provided the signal and noise occupy different portions of time-frequency space, the absence of cross-terms in equation 2 potentially allows for the 


\section{Filter coefficients for JCNB-SMNB}

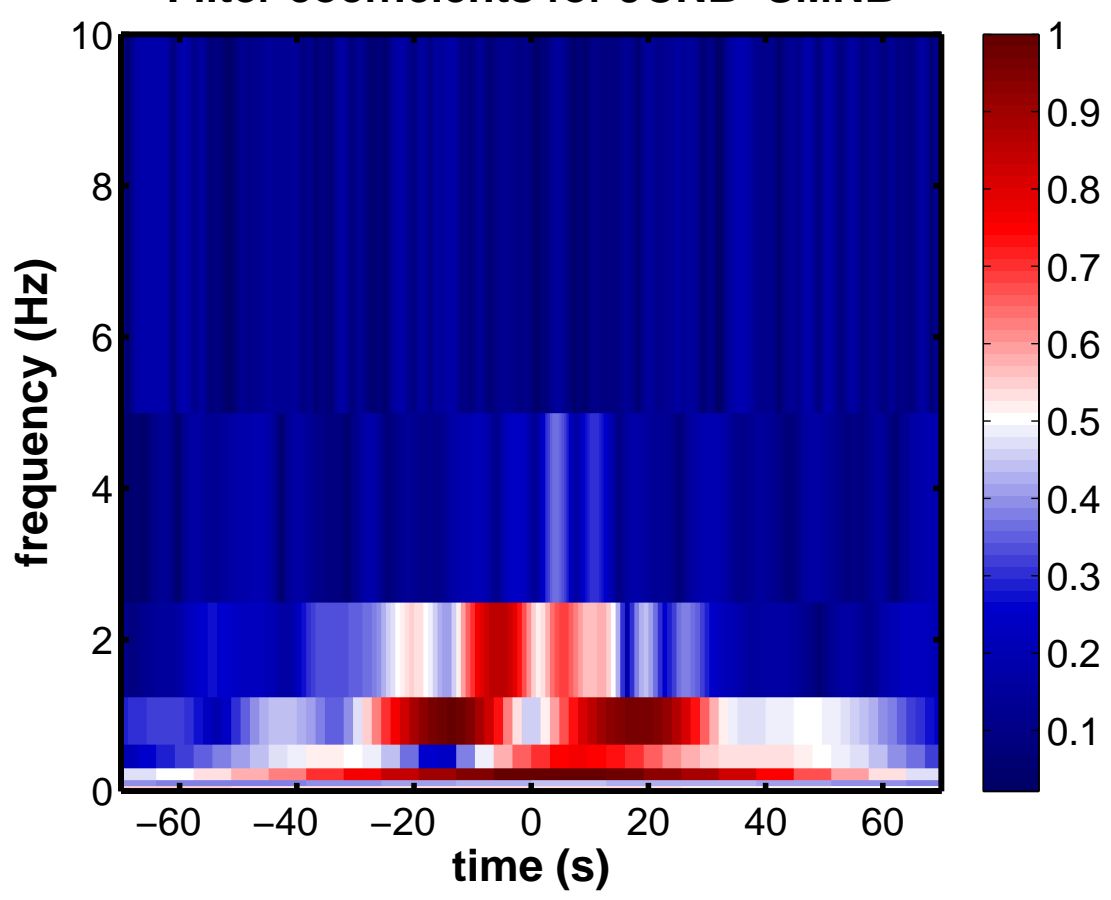

Figure 3: The absolute value of the coefficients of DOST basis vectors for the reference signal for JCNB-SMNB with each number coefficient plotted at its appropriate patch of time-frequency space. The colorscale ranges from dark blue - representing zero amplitude - to dark red for the highest amplitudes.

noise cancellation. We shall exploit this property in section 3.2.

\subsection{Construction of the Self-Filter}

To construct a denoising filter based on the data itself we use the linearity property of the S-transform, as shown in equation 2. The challenge is to identify the signal $g(t)$ when only the noisy timeseries $x_{i}(t)$ are measured. Suppose we have $M$ records of the same timeseries, $x_{i}(t)$. When we average over all these $x_{i}(t)$, and assume a random noise, the noise part of the record should tend to 0 as the number of records approaches $\infty$, and only the signal remains. We can see that with equation (2) the S-transform of the timeseries becomes:

$$
\langle S\{x\}(\tau, f)\rangle=S\{g\}(\tau, f)+\frac{1}{M} \sum_{k=1}^{M} S\left\{n_{k}\right\}(\tau, f)
$$

where $\langle$.$\rangle represents a normalized sum over the signals \frac{1}{M} \sum_{i=1}^{M}$. The second term in equation 3 disappears as $M \rightarrow \infty$. 
We can now construct a phase coherence filter $F$, inspired by the method used in Schimmel and Paulssen (1997), using the 'cleanest' possible signal $x(t)$. The signal $x(t)$ is decomposed into its DOST basis vectors $x_{k}$. The normalized, complex values of these basis vectors are stacked:

$$
F(\tau, f)=\left|\frac{1}{N} \sum_{k=1}^{N} \frac{S\left\{x_{k}\right\}(\tau, f)}{\left|S\left\{x_{k}\right\}(\tau, f)\right|}\right|^{\nu},
$$

The parameter $\nu$ can be used to control the strength of the filter. In this paper, $\nu=0.5$.

In this case, the reference stack takes the role of the clean signal $x(t)$ used in equation (3) and is used to construct the filter for each station pair. Each daily cross-correlation is then S-transformed, filtered according to:

$$
\int_{-\infty}^{\infty} S\left\{x_{i}\right\}(\tau, f) F(\tau, f) d \tau=X_{i}(f),
$$

and finally transformed back to the time-domain.

A drawback of the analytical S-transform (equation 1) is that it is computationally expensive to calculate. In practice, we apply the DOST transform to our signals in order to gain on computing time.

\section{Method: Doublets \& Stretching}

Two processing techniques have been proposed in the literature to estimate relative velocity changes $d v / v$ in the coda of the correlations. The first one, the doublet technique, was developed by Poupinet et al. (1984). It is also known as cross-spectral moving-window technique (CSMWT, Frechet et al. (1989)), and in practice computes the phase shift between records for consecutive, overlapping time windows $\Delta t$. For a given window $\Delta t$, the time shift $\delta t$ is assumed to be constant and is estimated in the frequency domain by measuring the Fourier cross-spectrum phase. This estimator uses an accurate, unbiased Wiener filter technique (Jenkins and Watts (1969)) and produces an estimate whose confidence interval is controlled by the coherence values in the frequency band used for the analysis. The method can then measure arbitrary time-shifts between two records with enough similarity (or coherence). It is consequently possible to represent the time-shift $\delta t$ versus time $t$ in the coda. The actual velocity variation is simply the average slope of $\delta t(t): d v / v=-\delta t / t$. The key parameter in this analysis is the Fourier transform window length $\Delta t$, which is the time-window over which the time-delay is measured. On one hand, large time windows include more data, thus averaging down the fluctuations of $\delta t$ due to noise. This results in more accurate measurements. On the other hand, the approximation that the time-shift is constant within the given time-window is getting more erroneous when the window length is increased. The choice of $\Delta t$ is therefore 
a trade-off between time-shift accuracy, and the time resolution between two consecutive measurements.

This processing found remarkable applications in seismology, including recent developments in ambient noise seismology (Brenguier et al. (2008b,a)). Another idea (Lobkis and Weaver (2003); Sens-Schönfelder and Wegler (2006)) is to interpolate the coda at times $t(1-\varepsilon)$ with various stretching factors $\varepsilon$. The actual relative velocity change is the stretching factor $\varepsilon_{0}=d v / v$ that maximizes the cross-correlation coefficient:

$$
X(\varepsilon)=\frac{\int_{t_{1}}^{t_{2}} h[t(1-\varepsilon)] h_{0}[t] d t}{\sqrt{\int_{t_{1}}^{t_{2}} h^{2}[t(1-\varepsilon)] d t \cdot \int_{t_{1}}^{t_{2}} h_{0}^{2}[t] d t}},
$$

where $t_{1}$ and $t_{2}$ the start and end time of the coda used, respectively.

One drawback is that this latter processing assumes a linear behavior $\delta t$ versus $t$, equivalent to a homogeneous relative velocity change $d v / v=\varepsilon$. This is sometimes not the case in complex media. But this technique has also a noticeable advantage: whole data is processed at once, which is found to result in a more stable, and thus more precise, estimation of $d v / v$. In particular, we showed from laboratory experiments that the stretching technique is more adapted to data with low signal-to-noise ratio (Hadziioannou et al. (2009)). It was also found very useful for noisy seismic correlations on the moon (Sens-Schönfelder and Larose (2008)). Another interesting feature of the stretching technique is to provide the remnant coherence $X(\varepsilon)$. This coherence indicates if noise sources are stable or changing over the period of observation of interest: $X \sim 1$ means absolutely stable sources, $X<<1$ means that sources locations are changing. $X$ also indicates the quality of the $\varepsilon$ estimation (see section 7). In the case of Parkfield, we end up with a $d v / v$ resolution of $10^{-3}$ for measurements on 1 day stacks, and up to $2 \cdot 10^{-4}$ for 30 day stacks (section 7 ).

\section{Results}

The adaptive filter is tested with the two methods (doublets and stretching) for retrieving velocity variations detailed in section 4 . Both analyses are done on cross-correlations bandpassed for $\left[\begin{array}{ll}0.1 & 0.9\end{array}\right] \mathrm{Hz}$.

\subsection{Velocity Variations from Doublets}

In the doublet analysis the time shift $\delta t$ is measured for 100 windows of $\Delta t=10 \mathrm{~s}$, which are shifted by 1 second. A slope is then fitted along the measurements for time windows ranging from -120 s to -20 s and +20 s to +120 s in the cross-correlation signal. The window from -20 s to $20 \mathrm{~s}$ is excluded, in order to minimize effects from the direct waves. 


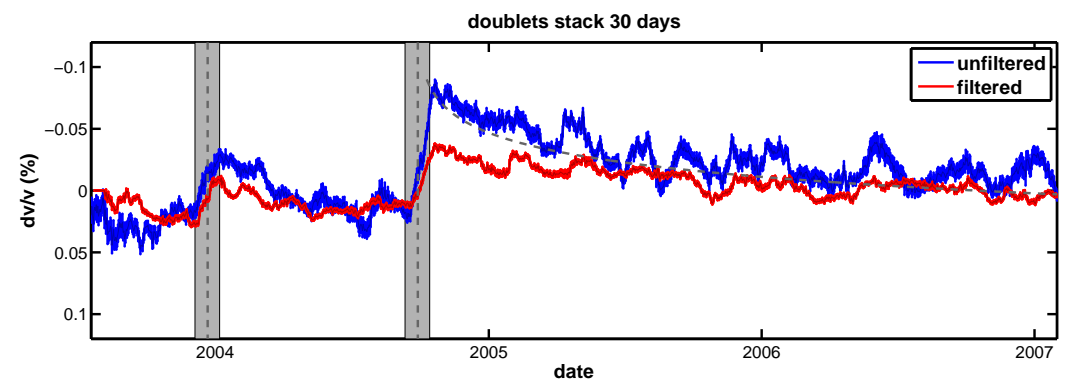

Figure 4: $d v / v$ using the doublet method, for a stack of 30 days. In blue: using the original data, in red: after applying the adaptive filter. The dashed line represents displacement measurements along the fault measured at the pomm GPS station (figure 1). The first vertical dashed line indicates the date of the San Simeon earthquake, the second that of the Parkfield earthquake. The extent of the stack used is shown as a shaded gray area.

Figure 4 shows the relative velocity changes obtained with the doublet method on 30 day stacks (which slide by 1 day), before (blue) and after (red) application of the adaptive filter. We can see that while the dispersion of the measurements is reduced, as seen by the reduction of the errorbars, the overall values of velocity change measured are reduced. This follows from the way the doublet method retrieves the time lag in the frequency domain (Poupinet et al. (1984)). The delay of each small window of the signal is measured by fitting a line to the phase-frequency behavior in the cross-spectrum of the signal and its reference. In Figure 3, the absolute value of each of the $N$ DOST basis vectors is portrayed on its corresponding time-frequency space. In a way, it represents the absolute value of the DOST basis function for the reference signal of station pair JCNB-SMNB. In this figure, it is visible that the adaptive filter applies different filtering coefficients for different frequencies, which affects this linear phase-frequency behavior. Since the doublet method measures the time delay by fitting a linear regression to the phase-frequency, this way of filtering interferes with the measurements. This lower amplitude of the $d v / v$ is a disadvantage of the adaptive filter when it is used in combination with the doublet technique. However, in some cases it can be useful to use the filter to increase the SNR and thereby the temporal resolution. This way, it is possible to pinpoint the date at which a change occurred, even if the amplitude is wrong. For stacks shorter than 10 days, the SNR of the signals become too small to apply the doublet method, even after filtering. 

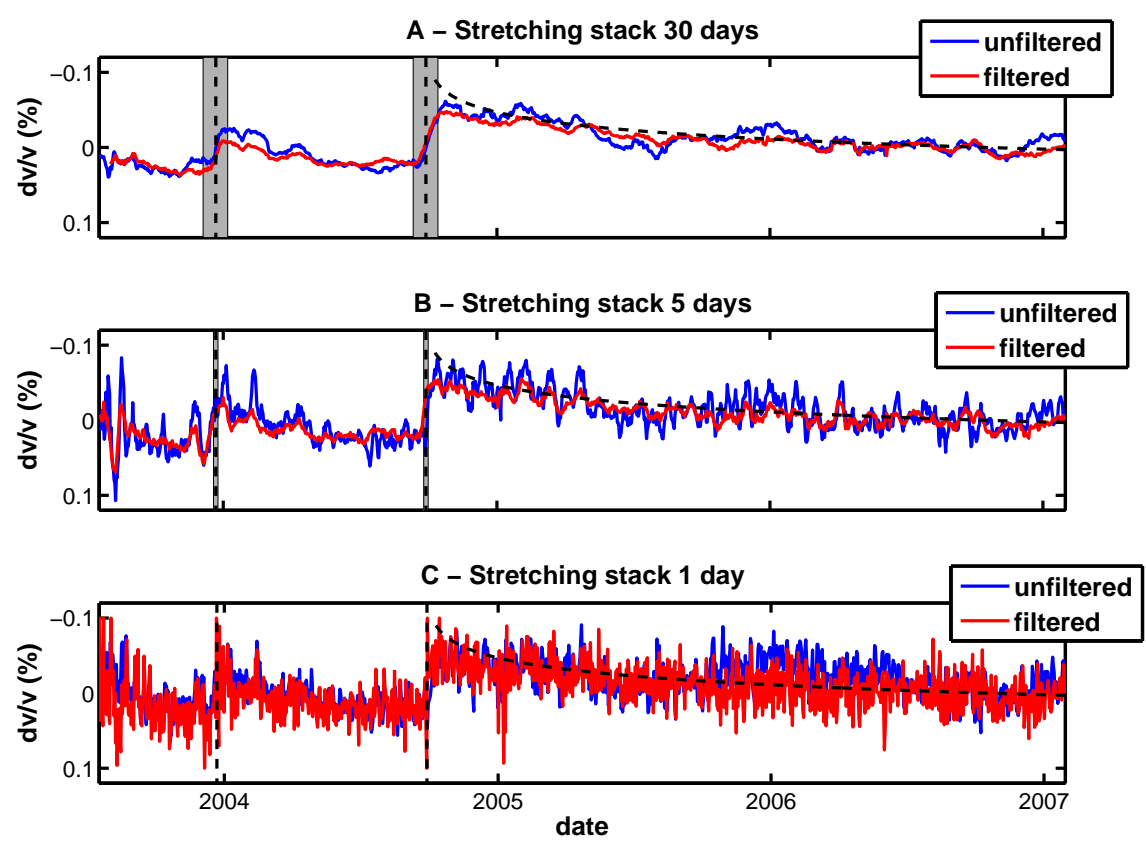

Figure 5: $d v / v$ using the stretching method, for a stack of 30 days (A), 5 days (B) and 1 day (C). In blue: using the original data, in red: after applying the adaptive filter. The black dashed line represents displacement measurements along the fault measured at the pomm GPS station (figure 1). The first black dashed line indicates the date of the San Simeon earthquake, the second that of the Parkfield earthquake. The shaded gray areas around the dashed lines show the extent of the stack used.

\subsection{Velocity Variations from Stretching}

The stretching technique measures the dilation of the signal using as much of the coda as possible (see equation 6 ) - in this case, from \pm 20 s to $\pm 120 \mathrm{~s}$. This renders the technique less sensitive to fluctuations in the correlations. In Figure 5a we show the velocity changes measured with the stretching method using 30 day stacks, with and without application of the filter. Again, we remark a reduction of fluctuations after applying the filter. However, unlike in the case of the doublet method, the filter does not degrade the amplitude of the variations measured. In Figure 5b, we observe the same with a stack of 5 days. Note that the postseismic behavior is still consistent with the postseismic slip as measured by Freed (2007) from GPS measurements ${ }^{4}$. Another improvement by the filter can be seen when re-

\footnotetext{
${ }^{4}$ More information concerning the USGS GPS network at Parkfield can be found at http://earthquake.usgs.gov/monitoring/edm/parkfield/continuous.php
} 


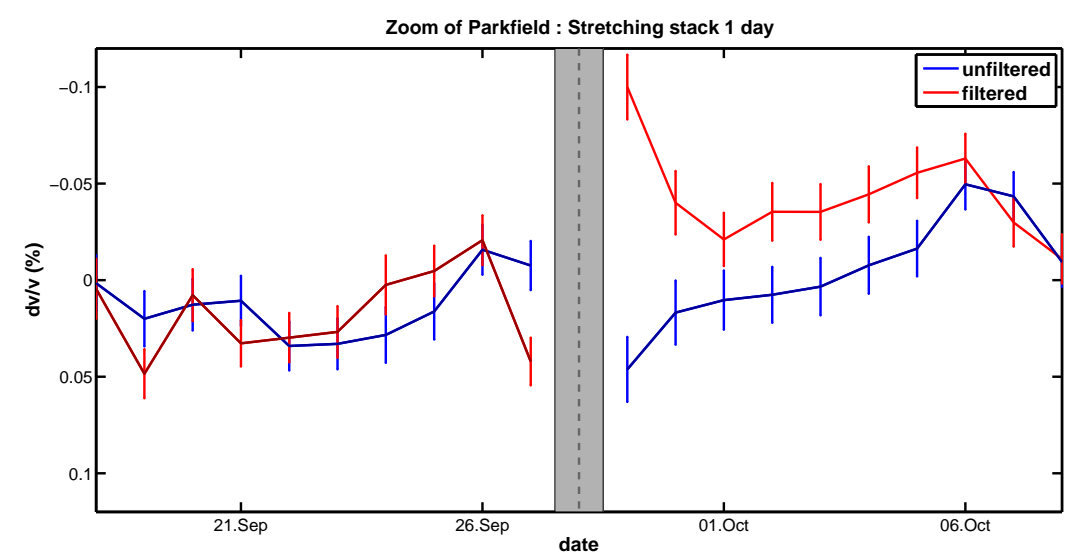

Figure 6: $d v / v$ using the stretching method, for a stack of 1 day, zoom around Parkfield earthquake (28 September, 2004). In blue: using the original data, in red: after applying the adaptive filter. The gray shading represents the day of the earthquake. Due to the many aftershocks this day interfering with the background noise, the $d v / v$ measurement is unreliable. The points for the day of the earthquake are therefore not plotted. The errorbars are described in section 7 .

ducing the stack length to only 1 day (Figure 5c). Here, when using the unfiltered signal, the velocity drops associated with the San Simeon and Parkfield earthquakes can be observed, but they become much clearer in the case of the filtered signal. This is especially apparent when we look closely at the velocity behavior around the date of the Parkfield earthquake (Figure 6). The errorbars shown in this figure are described in section 7 . The measurements from the filtered signals show that the velocity one day after the earthquake has dropped to a lower value, and recovers slowly.

Note that the ambient noise on the day of the earthquake is altered by numerous aftershocks and the mainshock itself. After elimination of these parts of the noise, not much data remains to work with on that day, thus producing an unreliable correlation signal. There also appears to be a velocity increase one day before the event, however, we would be very hesitant to claim a precursor based on this single data point. From these observations (Figure 6), we can safely conclude that the velocity drop we measure has its maximum at the time of the Parkfield earthquake (Rubinstein and Beroza (2005)).

Stretching, especially in combination with the adaptive filter to remove as much noise from the signals as possible, allows to greatly improve the temporal resolution of the measurements. 


\section{Noise Source Location from Beamforming}

After the previous discussion on the improvement of the temporal resolution of the $d v / v$ measurements, we study if the observed fluctuations in the velocity can be explained by the change in noise source distribution. We observe on the example of cross-correlations of couple JCNB-SMNB (Figure 2 , center) that the direct waves are sensitive to seasonal variation, probably due to variations in the noise source distribution. According to the theory (Gouédard et al. (2008), Froment et al. (2010), Paul et al. (2005)), we expect any strongly directive and varying part of the noise field to influence the Green function reconstruction negatively and thus alter the $d v / v$ measurement.

In order to establish if any such strong moving sources exist around the Parkfield array, we investigate the structure and evolution of the ambient noise field. To do so, we perform plane wave beamforming (as described in Roux (2009)) on 1-day segments of ambient noise in the [0.1 0.2] Hz frequency band which contains the secondary microseism peak. Figure 7 shows an example of the angular-speed distribution of the background noise on this frequency interval.

We observe that the ambient noise field at Parkfield is very directive and dominated by a component propagating to $\sim 50^{\circ}$ clockwise from North with an apparent velocity around $2500 \mathrm{~m} / \mathrm{s}$. This means that it consists mostly of surface waves originating from the direction of the Pacific Ocean, in agreement with results from similar analyses by Roux (2009).

We track the azimuthal variation of the most prominent maximum in Figure 8a. In order to quantify the temporal change of the incident direction of all noise sources, not only the strongest, we define a correlation of the noise incident direction. This is evaluated by looking at the daily coherence of the beamformer output with respect to the output averaged over the three years of data (Figure $8 \mathrm{~b}$ ).

Do the azimuthal variations of the dominant source, or variations of the noise field coherence, reflect on the $d v / v$ measurements? In Figure 8 , we have plotted the azimuthal variation of the strong source at $[0.10 .2] \mathrm{Hz}$, as well as the noise field coherence (a and $\mathrm{b}$ ) along with the $d v / v$ measurements and the correlation function coherence (c and d).

From Figure 8a, some seasonal variations in the azimuth of the beamformer output is visible, reminiscent of the variations seen on the cross-correlations in Figure 2. However, one can see that the change in noise direction does not influence the $d v / v$ measurements directly. On the other hand, the change in noise field structure, expressed as a decoherence in Figure 8b, does relate to a change in correlation waveform (Figure 8d). As seen in section 4, this decrease in waveform coherence as the source distribution changes, is expected. In some cases, such as in the beginning of 2005, a strong change in noise sources has even led to more fluctuations in the $d v / v$ measurements. 


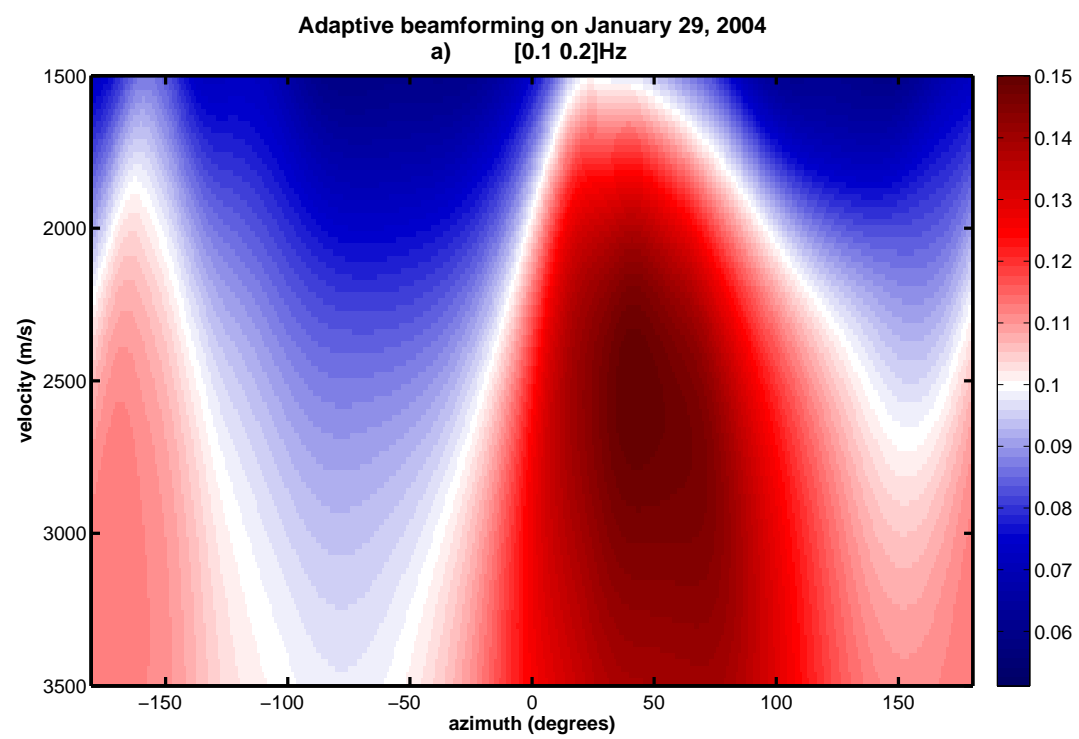

Figure 7: Beamforming output representing the angle-versus-velocity ambiguity surface at $\left[\begin{array}{ll}0.1 & 0.2\end{array}\right] \mathrm{Hz}$. Azimuth in degrees clockwise from North.

This effect of decreased waveform coherence on the measurements is included in the expected rms fluctuations, as can be seen in section 7 .

In conclusion, a change in noise wavefield incidence does temporarily result in a noisier $d v / v$ measurement. Since such a change is also visible as a decoherence of the waveform these periods of time can be detected and treated accordingly.

\section{$7 \quad$ Error calculation}

The precision with which a dilation coefficient $\epsilon$ is retrieved depends on the strength of correlation between two signals. Weaver et al. (2011) derived an expression to predict the fluctuations in dilation coefficient due to e.g. changes in the noise source, in the case where the velocity in the medium has not changed.

The goal is to evaluate the precision with which wave speed changes can be evaluated. To do this we consider the case in which two waveforms differ only by noise so that the actual relative dilation, without noise, is zero. We then look for the apparent (non-zero in general) value of $\epsilon$ at which the corresponding $X$ in equation (6) achieves its maximum. Equation 7 gives the root mean square of this apparent, and erroneous, relative dilation. 
a)

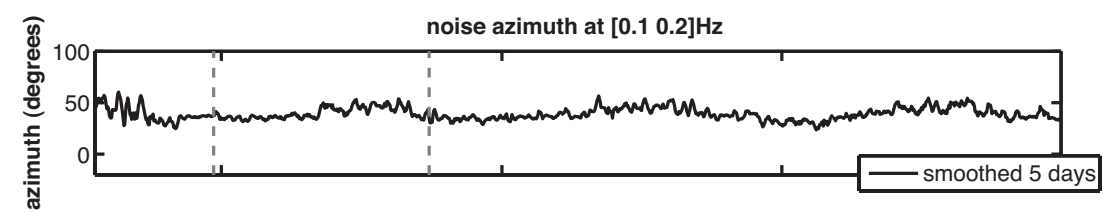

b)

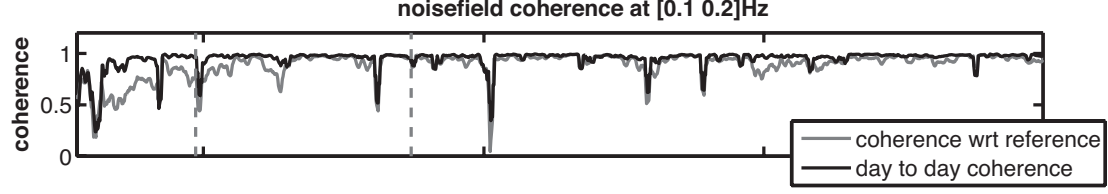

c)

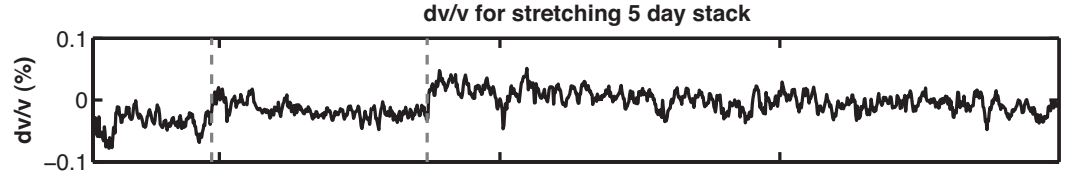

d)

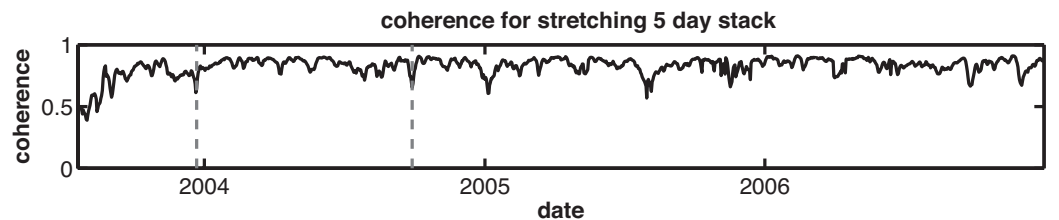

Figure 8: (a) The azimuth of the beamformer output maximum (in degrees clockwise from North) and (b) the beamformer output coherence, both at $\left[\begin{array}{ll}0.1 & 0.2\end{array}\right] \mathrm{Hz}$. (c) The velocity variations as well as (d) the correlation waveform coherence for a 5-day sliding stack. The gray vertical dashed lines represent the dates of the San Simeon earthquake and that of the Parkfield earthquake, respectively. 


$$
\operatorname{rms}(\epsilon)=\frac{\sqrt{1-X^{2}}}{2 X} \sqrt{\frac{6 \sqrt{\frac{\pi}{2}} T}{\omega_{c}^{2}\left(t_{2}^{3}-t_{1}^{3}\right)}},
$$

where $\epsilon$ is the dilation, $X$ the coherence of the waveform after dilation, between starting time $t_{1}$ and end time $t_{2} . T$ is the half period bandwidth, with $\omega_{c}$ the center frequency of the signal. In this study, values are typically $\omega_{c}=0.5, T=0.4$, with start time $t_{1}=20$ seconds and end time $t_{2}=120$ seconds. This leads to an $\operatorname{rms}(\epsilon)$ evaluated by:

$$
\operatorname{rms}(\epsilon)=2.6 \times 10^{-3} \frac{\sqrt{1-X^{2}}}{2 X},
$$

as detailed in Weaver et al. (2011). Since we average the measurement over 91 station pairs, this rms value is reduced by $\sqrt{91}$, which leads to a rms of the order of $8 \times 10^{-5}$ for a wavefield coherence of $X=0.8$. If the dilation measured exceeds this rms value, we can conclude that it is indeed due to a velocity change in the medium. We find that in the case of the Parkfield data, the velocity variations measured are larger than the predicted fluctuation value of $\epsilon$ for a correlation coefficient of $X$. This means that they are due to a local velocity change and not loss of coherence in the cross-correlation function.

\section{Discussion}

By improving the temporal resolution of our $d v / v$ measurements using an adaptive filter and the stretching technique, we have shown that the velocity drop observed at Parkfield is coseismic. The increase in temporal resolution obtained in this paper allows us to track velocity changes immediately after the event.

Similar, albeit stronger, coseismic velocity drops have been observed and are generally associated with shallow damage in either the crust (Rubinstein and Beroza (2004), Sawazaki et al. (2009), Peng and Ben-Zion (2006), Niu et al. (2003)) or the fault zone ( $\mathrm{Li}$ et al. (2003)). These shallow effects are modeled by a strong drop in shear modulus (and thus velocity drop) very shortly after the mainshock, followed by a postseismic logarithmic healing curve.

The peak ground acceleration measured in the region surrounding the array is in the order of $0.65-1.3 g$ (Shakal et al. (2005)), so superficial damage is a possiblity. It could be argued that the velocity drop we observe is a result of this shallow damage. The lower amplitude of velocity drop - on the order of $0.1 \%$ instead of $30 \%$ - could be the result of averaging a localized effect over a larger volume, seeing as we measure the change with multiply scattered coda waves.

However, if the velocity drop we observe were due only to superficial damage, we would expect the drop amplitude to become higher as we measure in a 
shorter time window just following the event (1 day as opposed to 30 days). As seen in Figure 5, this is not the case.

In fact, the postseismic relaxation behaviour closely follows along-fault displacement deduced from GPS measurements (Freed (2007), Johanson et al. (2006)). This suggests the possibility that not shallow, but deeper, deformationrelated effects are involved here.

Apart from the velocity drop at the Parkfield event, some small velocity fluctuations remain. As shown in section 7, these fluctuations are larger than the error expected on $d v / v$, and should have a physical origin. Since our GF is not perfectly reconstructed, these fluctuations could be due to a change of the sources. In section 6 , we find no direct relation between the dominant noise direction and the $d v / v$ measurements (Figure 8). However, the amount of decorrelation of the cross-correlations does reflect the stability of the ambient noise wavefield.

\section{Conclusion}

By using an adaptive filter to rid the data of as much noise as possible, and by applying the stretching method to retrieve the velocity variation measurements, we are able to perform monitoring on cross-correlations of only 1 day of ambient noise. Thanks to this, we can show that the $0.1 \%$ velocity change observed at the Parkfield event is well above the measurement precision and precisely coseismic.

Furthermore, we show that slow seasonal variations in the orientation of the main noise sources do not affect the velocity change measurements directly. Sudden changes in the noise wavefield do relate to large fluctuations measured in $d v / v$.

\section{Acknowledgments}

This work was funded by the ANR JC08_313906 SISDIF grant, and by the ERC advanced grant WHISPER.

The authors thank F. Brenguier, P. Gouédard, N. Schaeffer, R. Weaver and N. Shapiro for fruitful discussions and useful remarks.

All the seismic data used in this study came from the Parkfield HRSN and were collected by the Berkeley Seismological Laboratory (BSL).

\section{References}

Baig, A., M. Campillo, and F. Brenguier, 2009: Denoising seismic noise cross correlations. J. Geophys. Res., 114. 
Brenguier, F., M. Campillo, C. Hadziioannou, N. Shapiro, R. M. Nadeau, and E. Larose, 2008a: Postseismic relaxation along the San Andreas fault at Parkfield from continuous seismological observations. Science, 321 (5895), 1478-1481.

Brenguier, F., N. Shapiro, M. Campillo, V. Ferrazzini, Z. Duputel, O. Coutant, and A. Nercessian, 2008b: Towards forecasting volcanic eruptions using seismic noise. Nature Geoscience, 1 (2), 126-130.

Frechet, J., L. Martel, L. Nikolla, and G. Poupinet, 1989: Application of the Cross-Spectral Moving-Window Technique (CSMWT) to the Seismic Monitoring of Forced Fluid Migration in a Rock Mass. Int. J. Rock Mech. Min. Sci., 26 (3-4), 221-233.

Freed, A., 2007: Afterslip (and only afterslip) following the 2004 Parkfield, California, earthquake. Geophys. Res. Lett., 34 (6).

Froment, B., M. Campillo, P. Roux, P. Gouédard, A. Verdel and R. Weaver, 2010: Estimation of the effect of non-isotropic distributed energy on the apparent arrival time in correlations. Geophysics, 75 (5), SA85-SA93.

Gerstoft, P. and T. Tanimoto, 2007: A year of microseisms in southern California. Geophys. Res. Lett., 34, L20 304.

Gouédard, P., P. Roux, M. Campillo, and A. Verdel, 2008: Convergence of the two-point correlation function toward the green's function in the context of a seismic-prospecting data set. Geophysics, 73 (6), V47-V53.

Hadziioannou, C., E. Larose, O. Coutant, P. Roux, and M. Campillo, 2009: Stability of Monitoring Weak Changes in Multiply Scattering Media with Ambient Noise Correlation: Laboratory Experiments. J. Acoust. Soc. Am., 125 (6), 3688-3695.

Jenkins, G. and D. Watts, 1969: Spectral Analysis and Its Applications. Emerson-Adams Press.

Johanson, I., E. Fielding, F. Rolandone, and R. Burgmann, 2006: Coseismic and Postseismic Slip of the 2004 Parkfield Earthquake from SpaceGeodetic Data. Bull. Seismol. Soc. Am., 96 (4 B), 269-282.

Landès, M., F. Hubans, N. Shapiro, A. Paul, and M. Campillo, 2010: Origin of deep ocean microseisms by using teleseismic body waves. J. Geophys. Res., 115.

Larose, E., P. Roux, M. Campillo, and A. Derode, 2008: Fluctuations of correlations and Green's function reconstruction: role of scattering. $J$. Appl. Phys., 103 (11), 114907-114907. 
Li, Y., P. Chen, E. Cochran, and J. Vidale, 2007: Seismic velocity variations on the San Andreas fault caused by the 2004 M6 Parkfield Earthquake and their implications. Earth Planets Space, 59 (1), 21-31.

Li, Y., J. Vidale, S. Day, D. Oglesby, and E. Cochran, 2003: Postseismic Fault Healing on the Rupture Zone of the 1999 M 7.1 Hector Mine, California, Earthquake. Bull. Seismol. Soc. Am., 93 (2), 854-869.

Lobkis, O. and R. Weaver, 2003: Coda-Wave Interferometry in Finite Solids: Recovery of P-to-S Conversion Rates in an Elastodynamic Billiard. Phys. Rev. Lett., 90 (25), 254302.

Niu, F., P. Silver, R. Nadeau, and T. McEvilly, 2003: Migration of seismic scatterers associated with the 1993 Parkfield aseismic transient event. $\mathrm{Na}$ ture, 426 (6966), 544-548.

Paul, A., M. Campillo, L. Margerin, E. Larose, and A. Derode, 2005: Empirical synthesis of time-asymmetrical Green functions from the correlation of coda waves. J. Geophys. Res., 110.

Peng, Z. and Y. Ben-Zion, 2006: Temporal changes of shallow seismic velocity around the Karadere-Düzce branch of the north Anatolian fault and strong ground motion. Pure and Applied Geophysics, 163 (2), 567-600.

Poupinet, G., W. Ellsworth, and J. Frechet, 1984: Monitoring Velocity Variations in the Crust Using Earthquake Doublets - An Application to the Calaveras Fault, California. J. Geophys. Res., 89 (NB7), 5719-5731.

Roux, P., 2009: Passive seismic imaging with directive ambient noise: application to surface waves and the san andreas fault in parkfield, ca. Geophys. J. Int., 179 (1), 367-373.

Roux, P., K. Sabra, P. Gerstoft, W. Kuperman, and M. Fehler, 2005: Pwaves from cross-correlation of seismic noise. Geophys. Res. Lett., 32, L19 303.

Roux, P., W. Kuperman, 2004: Extraction of coherent wavefronts from ocean ambient noise. J. Acoust. Soc. Am., 116(4), 1995-2003.

Rubinstein, J. and G. Beroza, 2004: Evidence for Widespread Nonlinear Strong Ground Motion in the MW 6.9 Loma Prieta Earthquake. Bull. Seismol. Soc. Am., 94 (5), 1595-1608.

Rubinstein, J. and G. Beroza, 2005: Depth constraints on nonlinear strong ground motion from the 2004 Parkfield earthquake. Geophys. Res. Lett., 32.

Rubinstein, J., N. Uchida, and G. Beroza, 2007: Seismic velocity reductions caused by the 2003 Tokachi-Oki earthquake. J. Geophys. Res., 112 (B5). 
Sabra, K. G., P. Gerstoft, P. Roux, W. A. Kuperman, and M. C. Fehler, 2005: Surface wave tomography from microseisms in southern california. Geophys. Res. Lett., 32, L14311.

Sanchez-Sesma, F. and M. Campillo, 2006: Retrieval of the Green's function from cross correlation: the canonical elastic problem. Bull. Seismol. Soc. Am., 96 (3), 1182.

Sawazaki, K., H. Sato, H. Nakahara, and T. Nishimura, 2009: Time-Lapse Changes of Seismic Velocity in the Shallow Ground Caused by Strong Ground Motion Shock of the 2000 Western-Tottori Earthquake, Japan, as Revealed from Coda Deconvolution Analysis. Bull. Seismol. Soc. Am., 99 (1), 352-366.

Schimmel, M. and H. Paulssen, 1997: Noise reduction and detection of weak, coherent signals through phase-weighted stacks. Geophys. J. Int., 130 (2), 497-505.

Sens-Schönfelder, C. and E. Larose, 2008: Temporal changes in the lunar soil from correlation of diffuse vibrations. Phys. Rev. E, 78 (4), 45601.

Sens-Schönfelder, C. and U. Wegler, 2006: Passive image interferometry and seasonal variations of seismic velocities at Merapi Volcano, Indonesia. Geophys. Res. Lett., 33, 1-5.

Shakal, A., Graizer, V., Huang, M., Borcherdt, R., Haddadi, H., Lin, K., Stephens, C. and Roffers, P., 2005: Preliminary analysis of strong-motion recordings from the 28 September 2004 Parkfield, California earthquake. Seis. Res. Lett., 76 (1), 27.

Shapiro, N. and M. Campillo, 2004: Emergence of broadband Rayleigh waves from correlations of the ambient seismic noise. Geophys. Res. Lett., 31 (7), 1615-1619.

Shapiro, N. , M. Campillo, L. Stehly and M. Ritzwoller, 2005: Highresolution surface-wave tomography from ambient seismic noise. Science, 307 (5715), 1615-1617.

Stockwell, R., 2007: A basis for efficient representation of the S-transform. Digital Signal Processing, 17 (1), 371-393.

Stockwell, R., L. Mansinha, and R. Lowe, 1996: Localization of the complex spectrum: The $\mathrm{S}$ transform. IEEE Transactions on Signal Processing, 44 (4), 998-1001.

Wapenaar, K., 2004: Retrieving the elastodynamic Green's function of an arbitrary inhomogeneous medium by cross correlation. Phys. Rev. Lett., 93, 254301. 
Weaver, R., C. Hadziioannou, E. Larose, , and M. Campillo, 2011: On the precision of noise-correlation interferometry. Geophys. J. Int., in press

Weaver, R. and O. Lobkis, 2004: Diffuse fields in open systems and the emergence of the Green's function(L). J. Acoust. Soc. Am., 116 (5), 27312734 . 